\title{
Complete Molecular Weight Profiling of Low Molecular Weight Heparins Using Size Exclusion Chromatography-Ion Suppressor-High Resolution Mass Spectrometry
}

Joseph Zaia ${ }^{\dagger, \ddagger, *}$, Kshitij Khatri ${ }^{\dagger}$, Joshua Klein ${ }^{\dagger, \neq}$, Chun Shao ${ }^{\dagger}$, Yuewei Sheng ${ }^{\dagger}$, and Rosa Viner§

${ }^{\dagger}$ Center for Biomedical Mass Spectrometry, Dept. of Biochemistry, Boston University Medical Campus, 670 Albany St., Boston, MA 02118, USA

‡Bioinformatics Program, Boston University

§Thermo Fisher Scientific, San Jose, California 95134, United States

*Corresponding author

Center for Biomedical Mass Spectrometry

Dept. of Biochemistry

Boston University Medical Campus

670 Albany St., Rm. 509

Boston, MA 02118

(v) 617-638-6762

(f) 617-638-6761

(e) jzaia@bu.edu

Supporting information methods p. 2

Supporting information figures p. 4 


\section{Supporting Information Methods}

\section{Materials}

The United States Pharmacopeia standard for enoxaparin sodium was purchased from Sigma Aldrich (St. Louis, MO). Dalteparin sodium as Fragmin was obtained from Pharmacia Corporation (Kalamazoo, MI). Stock solutions of enoxaparin sodium and dalteparin sodium were prepared at $10 \mathrm{nmol} / \mu \mathrm{L}$ in water. They were then desalted by dialysis against $50 \mathrm{mM}$ ammonium formate $(\mathrm{pH} 6.8)$ in methanol:water (20:80) using 500 Da molecular weight membranes (The Nest Group, Southborough, MA).

\section{SEC-IC-MS.}

Tandem Acquity UPLC BEH columns $(4.6 \mathrm{~mm} \times 150 \mathrm{~mm}$ and $4.6 \mathrm{~mm} \times 300 \mathrm{~mm}$, Waters Corp., Milford, MA) were used to separate the LMWH preparations using conditions similar to a published method ${ }^{12}$. The LC column was connected to a chemically regenerated ion suppressor (ACRS 500, 2 mm, Thermo Fisher Scientific/Dionex, San Jose, CA) for online desalting. The mobile phase contained $50 \mathrm{mM}$ ammonium formate $(\mathrm{pH} \mathrm{6.8)} \mathrm{in} \mathrm{methanol:water}$ (80:20). The constituents were eluted in $90 \mathrm{~min}$ at a flow rate of $75 \mu \mathrm{L} / \mathrm{min}$. The ion suppressor exchanged ammonium salts to their corresponding organic acids, thereby eliminating the problem of ammonium adduction of the saccharide ions. A solution of $100 \mathrm{mM}$ sulfuric acid was used to regenerate the suppressor. The suppressor effluent was connected to the standard ESI source of a Q-Executive Plus mass spectrometer (Thermo Fisher Scientific, San Jose, CA). The mass spectra were acquired using the negative ionization mode at resolution 75,000 and $\mathrm{m} / \mathrm{z}$ range 200-2000 Th with three microscans. The source parameters for the analyses were optimized to minimize sulfate loss and included a spray voltage of $3.8 \mathrm{kV}$, a capillary temperature of $280^{\circ} \mathrm{C}$, a sheath gas flow of 10 , and an S-lens RF level of 40.

\section{Data Deconvolution}


The acquired spectra were manually examined and averaged over retention time ranges using the Xcalibur software (version 3.0.63, ThermoFisher Scientific, San Jose, CA). The combined spectra were auto-deconvoluted by an in-house software, GAGdecon

(https://github.com/BostonUniversityCBMS/gagdecon. GAGdecon uses a database search algorithm for structural assignment at the MS level. It maps the isotopic distribution of theoretical species using a python implementation of the BRAIN (Baffling Recursive Algorithm for Isotopic distribution calculations) $)^{13,14}$ available at (https://github.com/BostonUniversityCBMS/brainpy) to the experimental data.

GAGdecon works by constructing a combinatorial database of GAG compositions, computing their theoretical monoisotopic mass at a range of user specified charge states. The composition includes the number of monosaccharide species: HexN, HexA, HexNAc, dHexA, ManN, as well as substituents and adducts: $\mathrm{Ac}, \mathrm{Na}, \mathrm{K}, \mathrm{Ca}$, and $\mathrm{Li}$. The disaccharide pattern was extrapolated from the user input GAG species, and the counts of each monosaccharide scale with chain length (degree of polymerization, $\mathrm{dp}$ ), which may either be a range, or a fixed value. GAGdecon can process either centroid or profile data, using a Lorentzian peak shape fit to calculate the centroids for profile peaks. Once the input spectra have been preprocessed, database search is carried out by searching each theoretical GAG composition and charge state, generating a theoretical isotopic cluster based on the elemental composition of the theoretical molecular composition, including the first $95 \%$ of the isotopic pattern. Each theoretical isotopic pattern was fitted to the peak list, producing a score of the form:

$$
G=2 \sum_{i} \frac{O_{i}}{\sum_{j} O_{j}} \log \left(\frac{T_{i} / \sum_{j} T_{j}}{O_{i} / \sum_{j} O_{j}}\right)
$$

$O_{i}$ is the abundance of the ith observed peak and $T_{i}$ is the abundance of the theoretical peak. Optimal fits minimize $G$. Each fit with a score below a parameterized threshold is reported to the user. 


\section{Complete Molecular Weight Profiling of Low Molecular Weight Heparins Using Size Exclusion Chromatography-Ion Suppressor-High Resolution Mass Spectrometry}

Joseph Zaia ${ }^{\dagger, \ddagger}{ }^{\star}$, Kshitij Khatri ${ }^{\dagger}$, Joshua Klein ${ }^{\dagger, \ddagger}$, Chun Shao ${ }^{\dagger}$, Yuewei Sheng ${ }^{\dagger}$, and Rosa Viner ${ }^{\S}$ ${ }^{\dagger}$ Center for Biomedical Mass Spectrometry, Dept. of Biochemistry, Boston University Medical Campus, 670 Albany St., Boston, MA 02118, USA

¥Bioinformatics Program, Boston University

$\S$ Thermo Fisher Scientific, San Jose, California 95134, United States

\section{Supporting Information Figures}
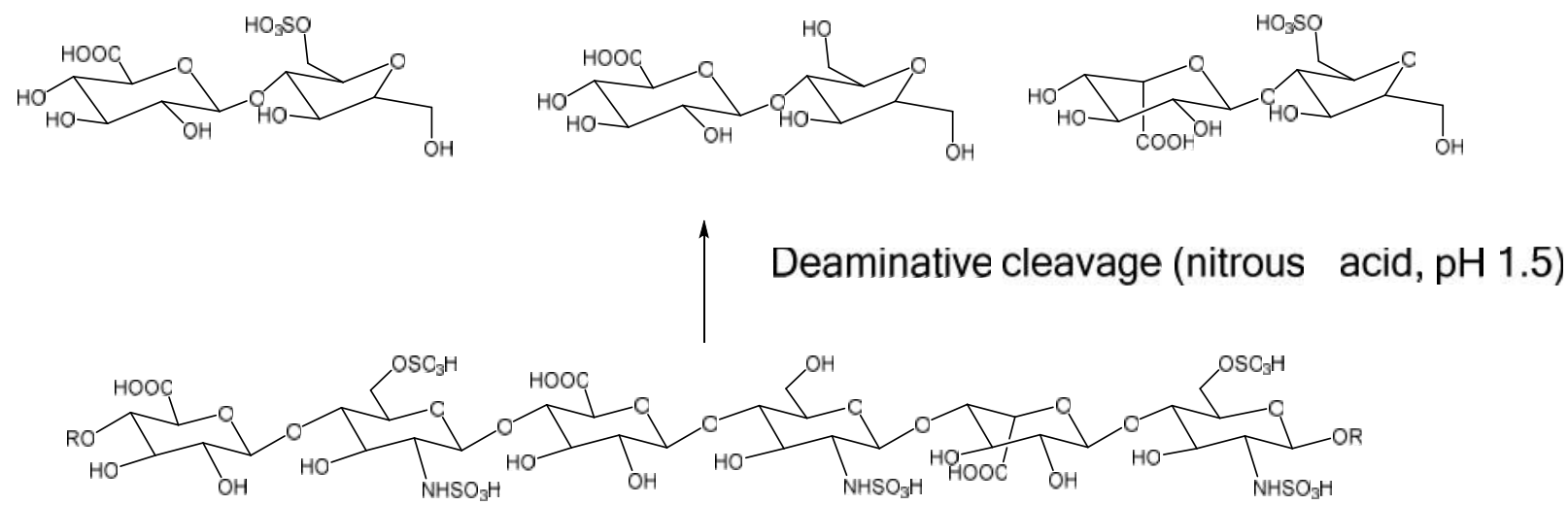

Ring contraction side reaction
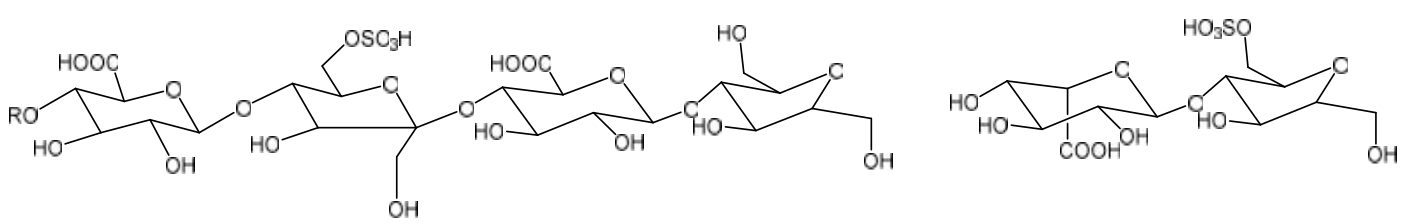

Figure S1. Top: deaminative cleavage of heparin using nitrous acid, $\mathrm{pH}$ 1.5. Bottom: ring contraction side reaction that occurs with deaminative cleavage, equivalent to loss of $\mathrm{NH}$ (Guo, Y. C.; Conrad, H. E. Anal Biochem 1989, 176, 96-104). 


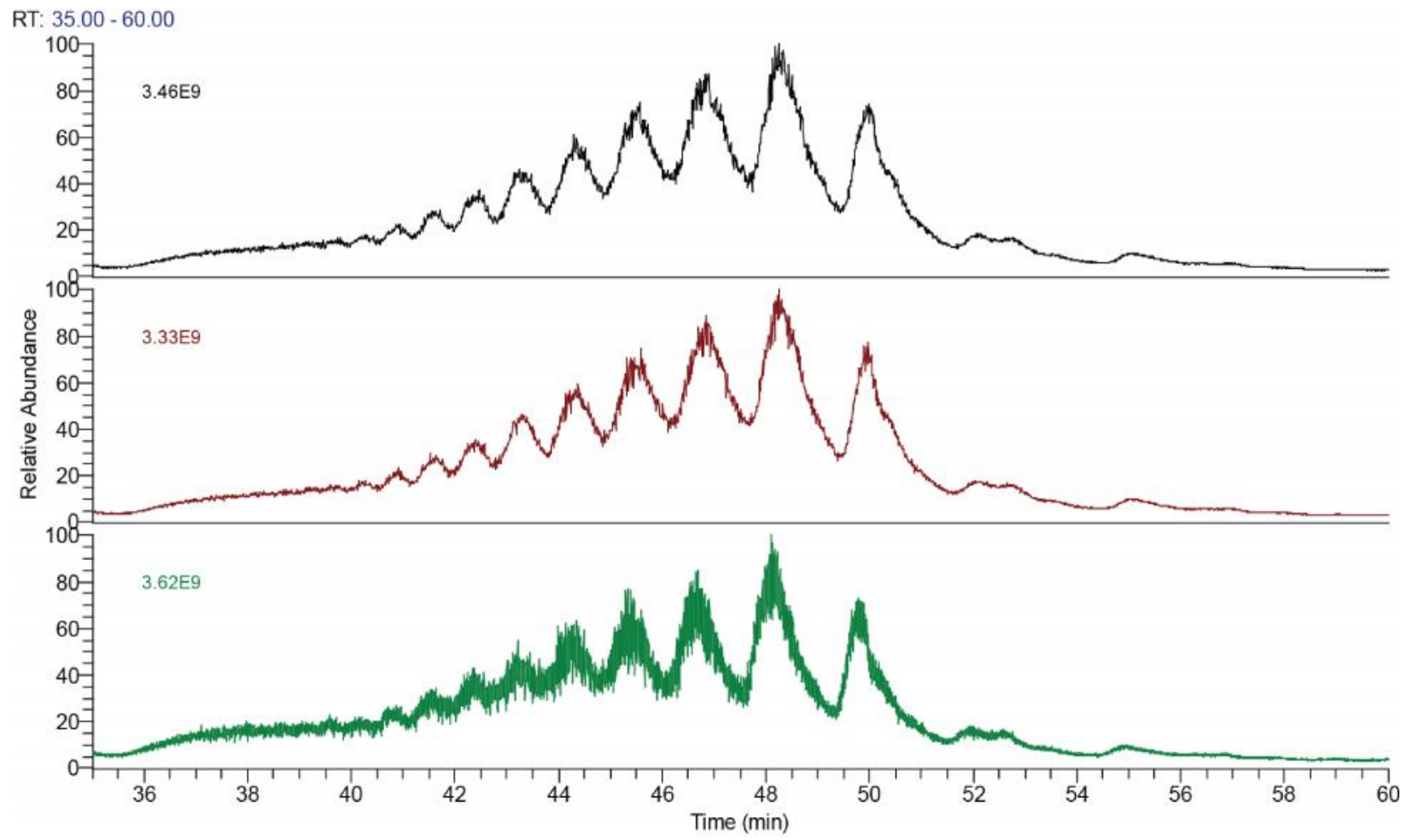

Figure S2a. Replicate SEC-IS-MS analysis of dalteparin, demonstrating reproducibility of chromatographic peaks and abundances. The top two SEC-IS-MS runs were acquired using an $\mathrm{m} / \mathrm{z}$ range of 200-2000. The lower run was acquired using an $\mathrm{m} / \mathrm{z}$ range of 200-3000, accounting for the difference in noise baseline. 


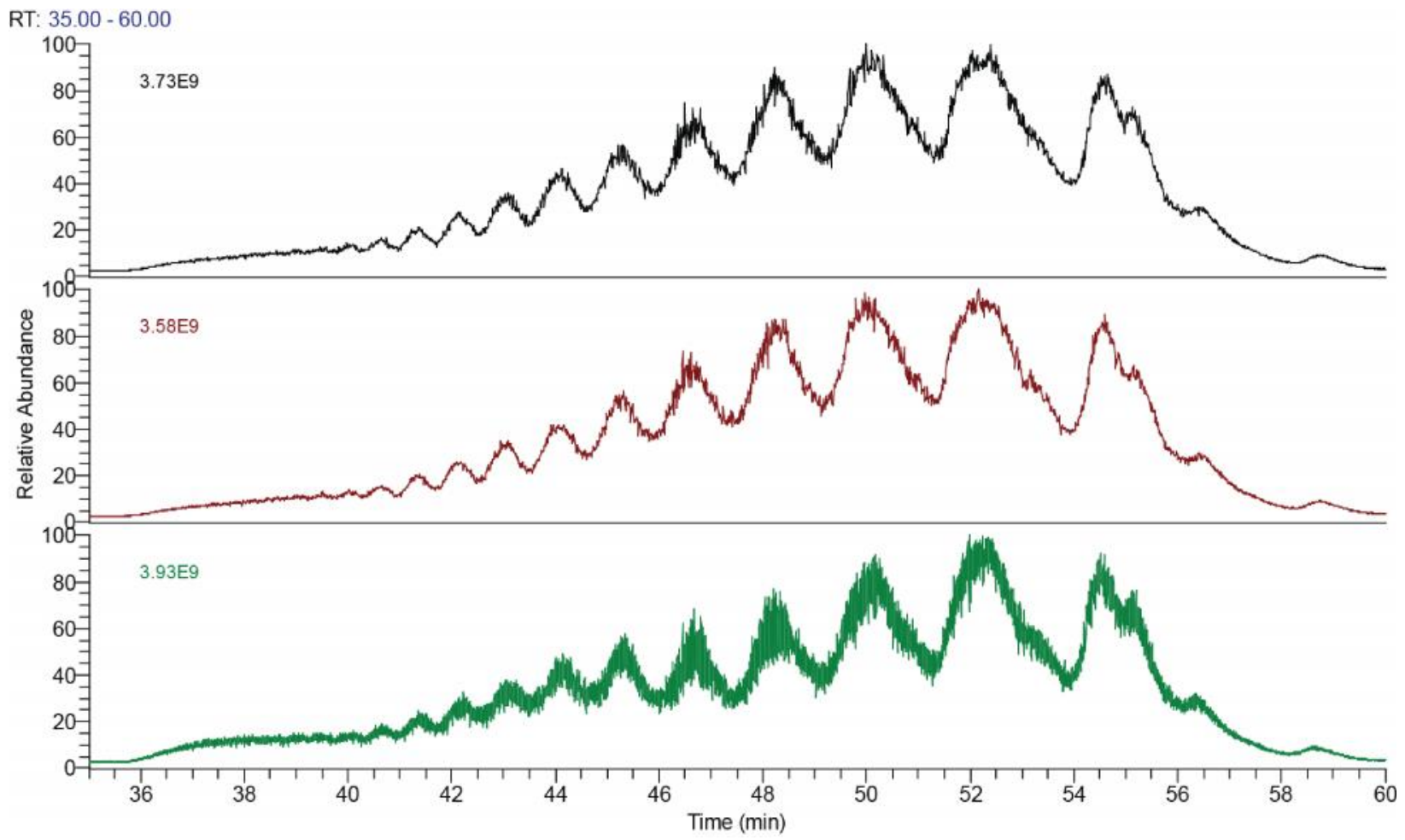

Figure S2b. Replicate SEC-IS-MS analyses of enoxaparin demonstrating reproducibility of chromatographic peaks and abundances. The top two SEC-IS-MS runs were acquired using an $\mathrm{m} / \mathrm{z}$ range of 200-2000. The lower run was acquired using an $\mathrm{m} / \mathrm{z}$ range of 200-3000, accounting for the difference in noise baseline. 
Figure S3. SEC-IS-MS compositional profiles for dalteparin saccharides. Ring contraction is indicated as $\mathrm{NH}$ in the plots. Higher confidence correlates with lower score. 
Composition Abundance Across Charge States

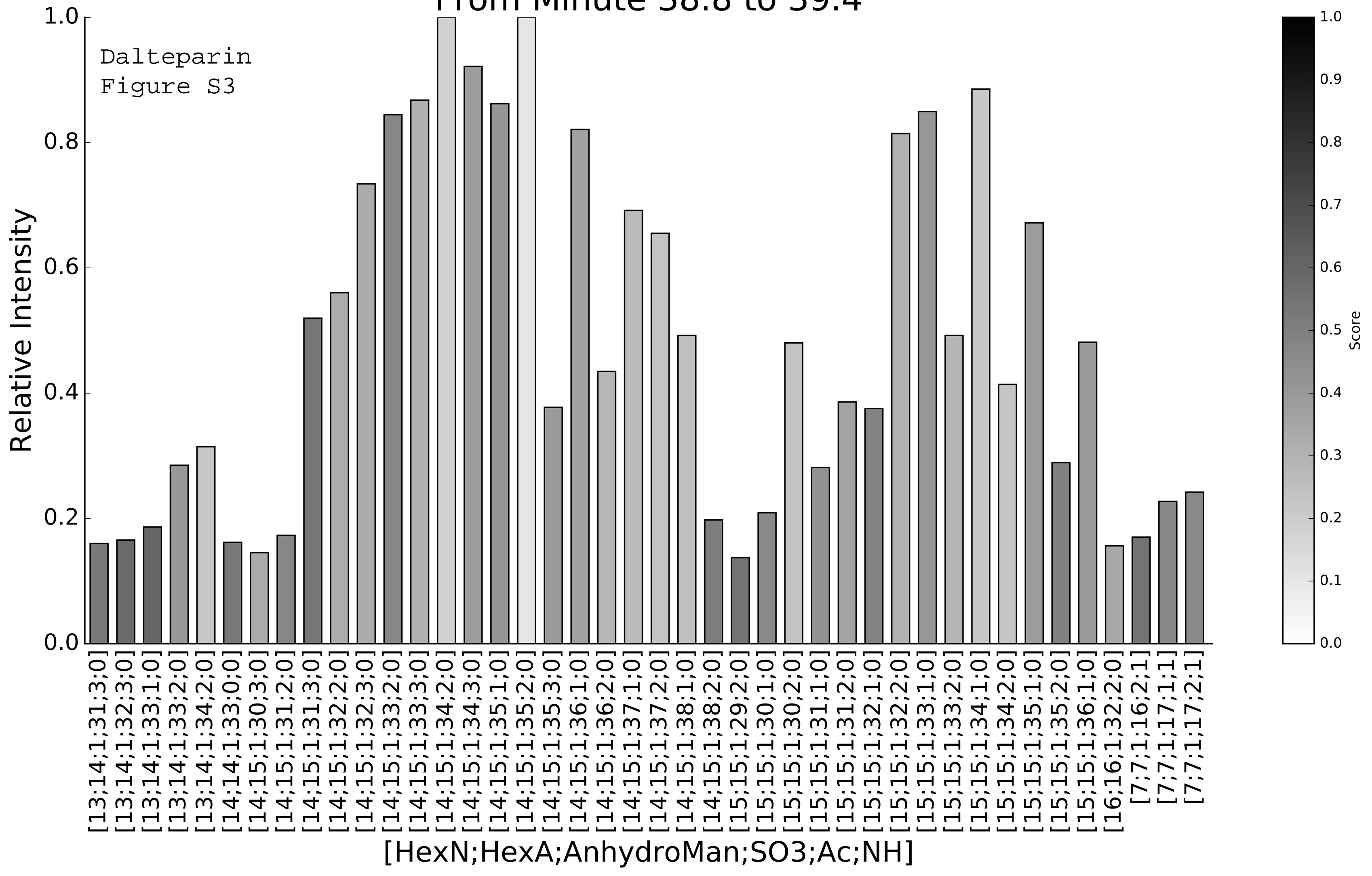


Composition Abundance Across Charge States

From Minute 39.4 to 39.8

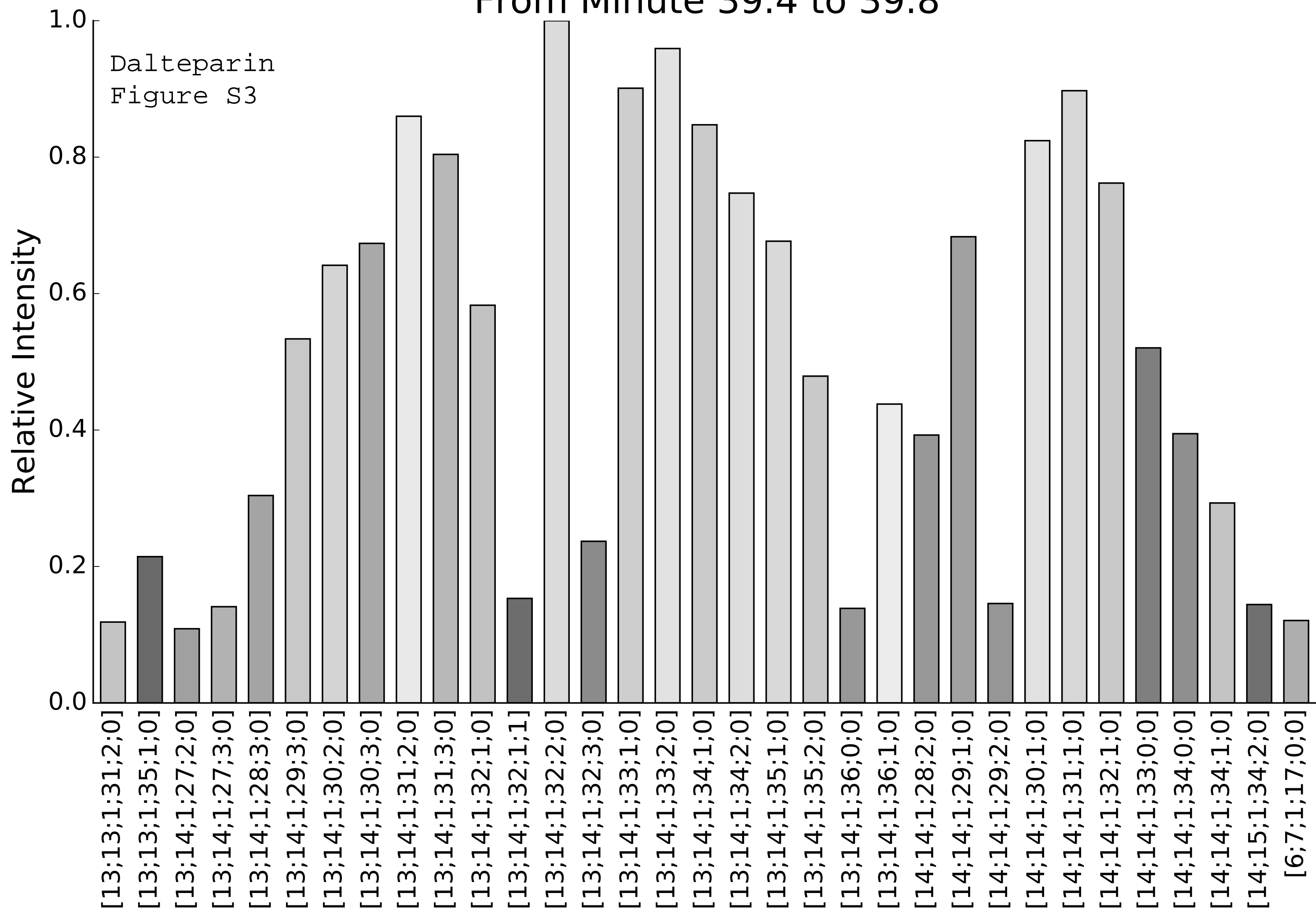

[HexN;HexA;AnhydroMan;SO3;Ac;NH] 


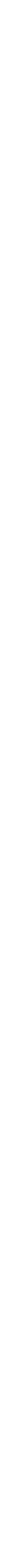


Composition Abundance Across Charge States

From Minute 40.4 to 41.1

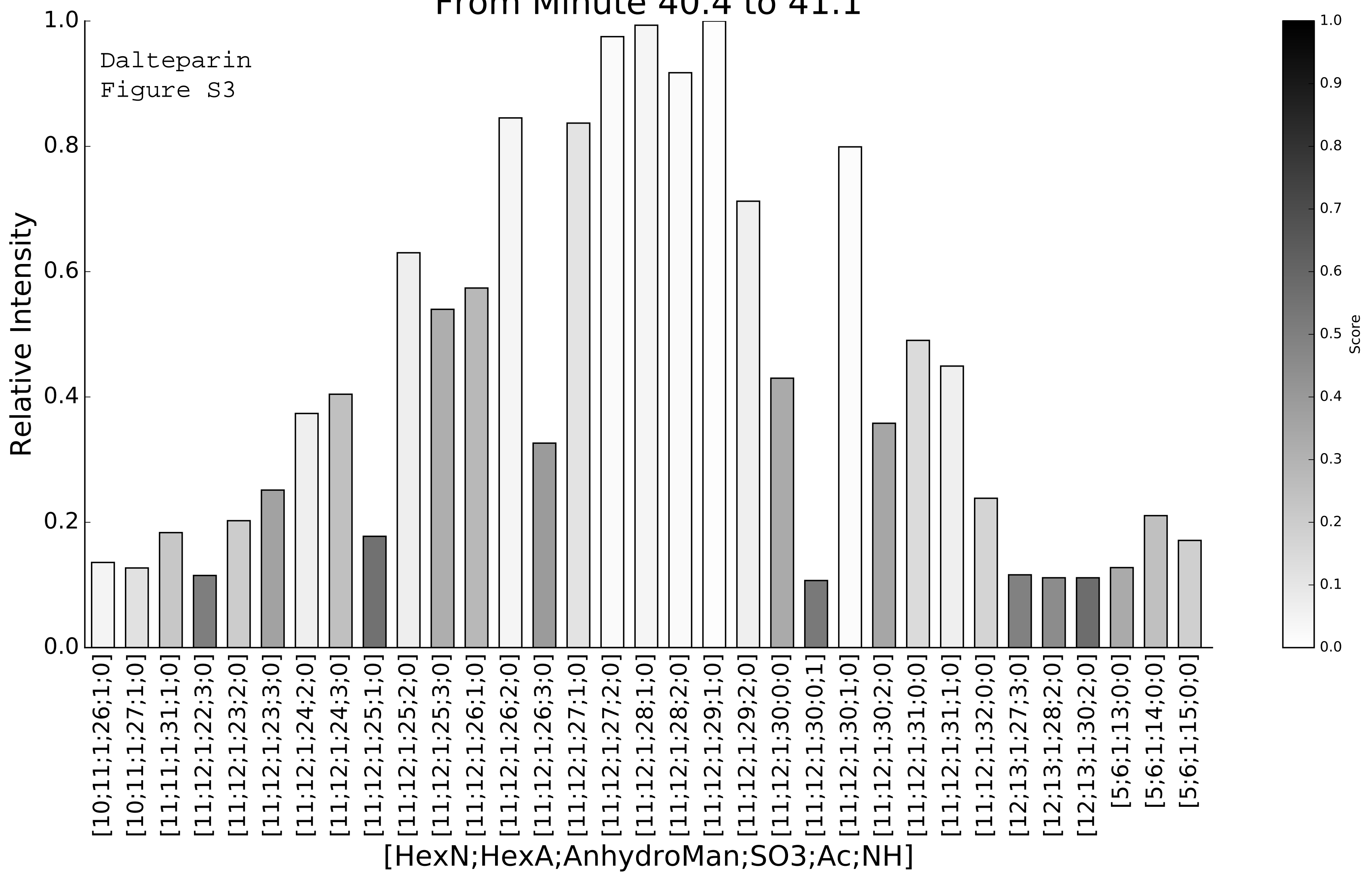


Composition Abundance Across Charge States

From Minute 41.0 to 41.9

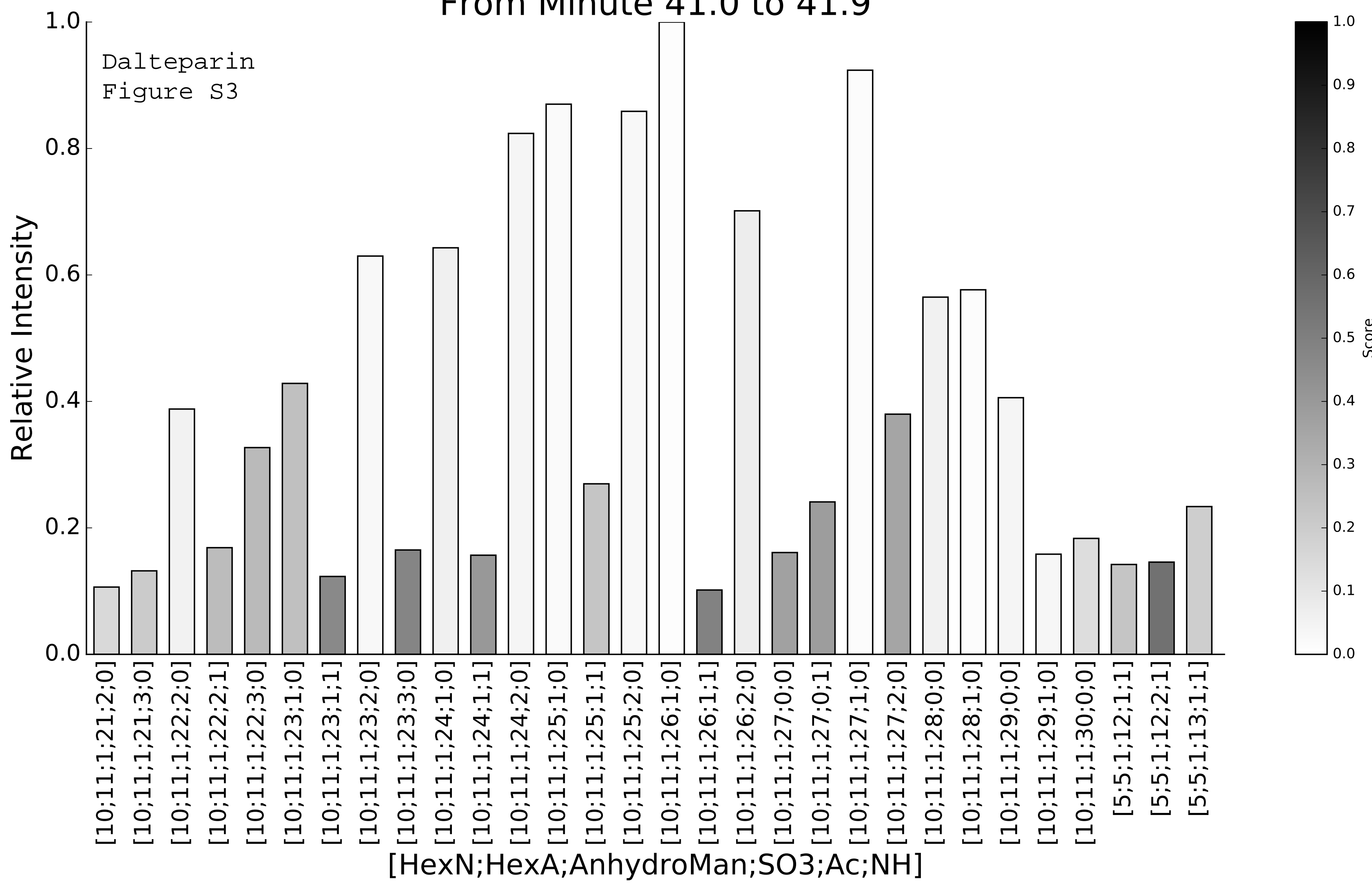


Composition Abundance Across Charge States

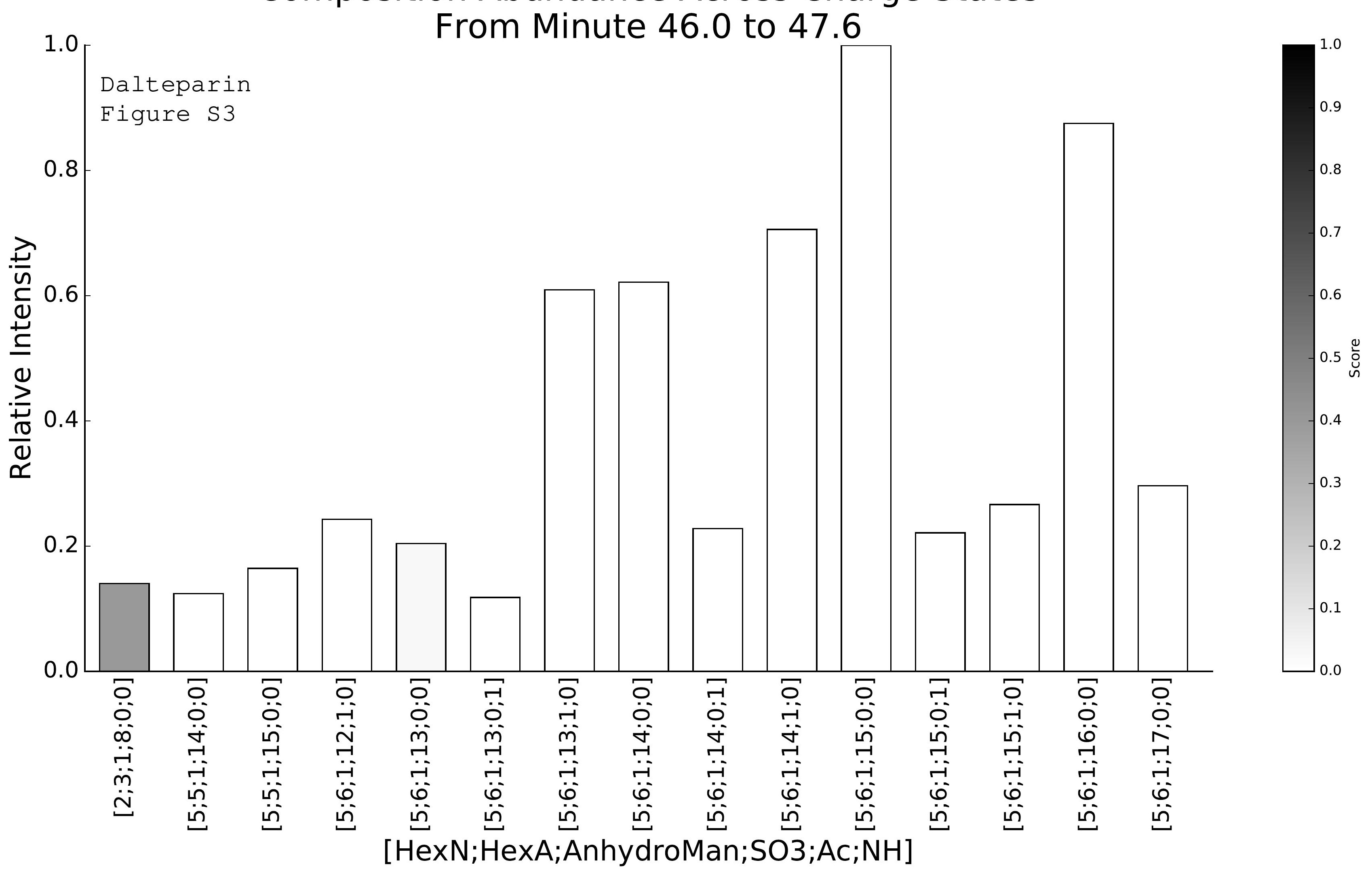


Composition Abundance Across Charge States

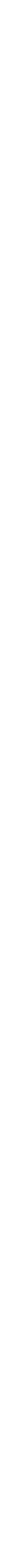


Figure S4. SEC-IS-MS compositional profiles for enoxaparin saccharides. The number of water losses is given as $\mathrm{H}_{2} \mathrm{O}$ in the plots. Higher confidence correlates with lower score. 


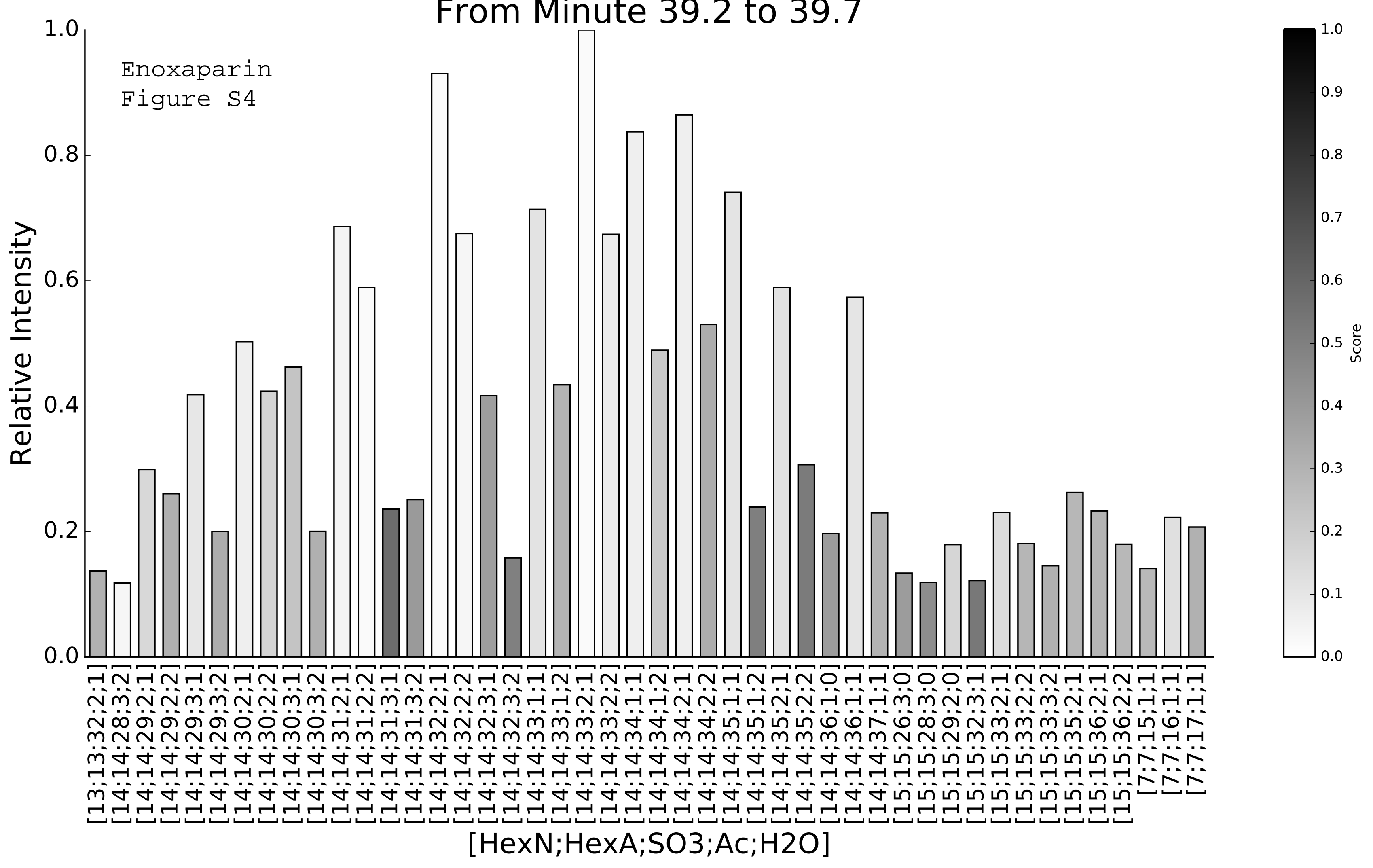




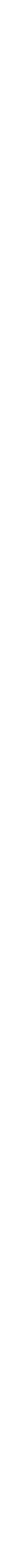




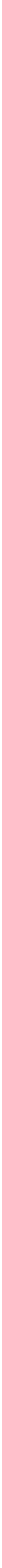




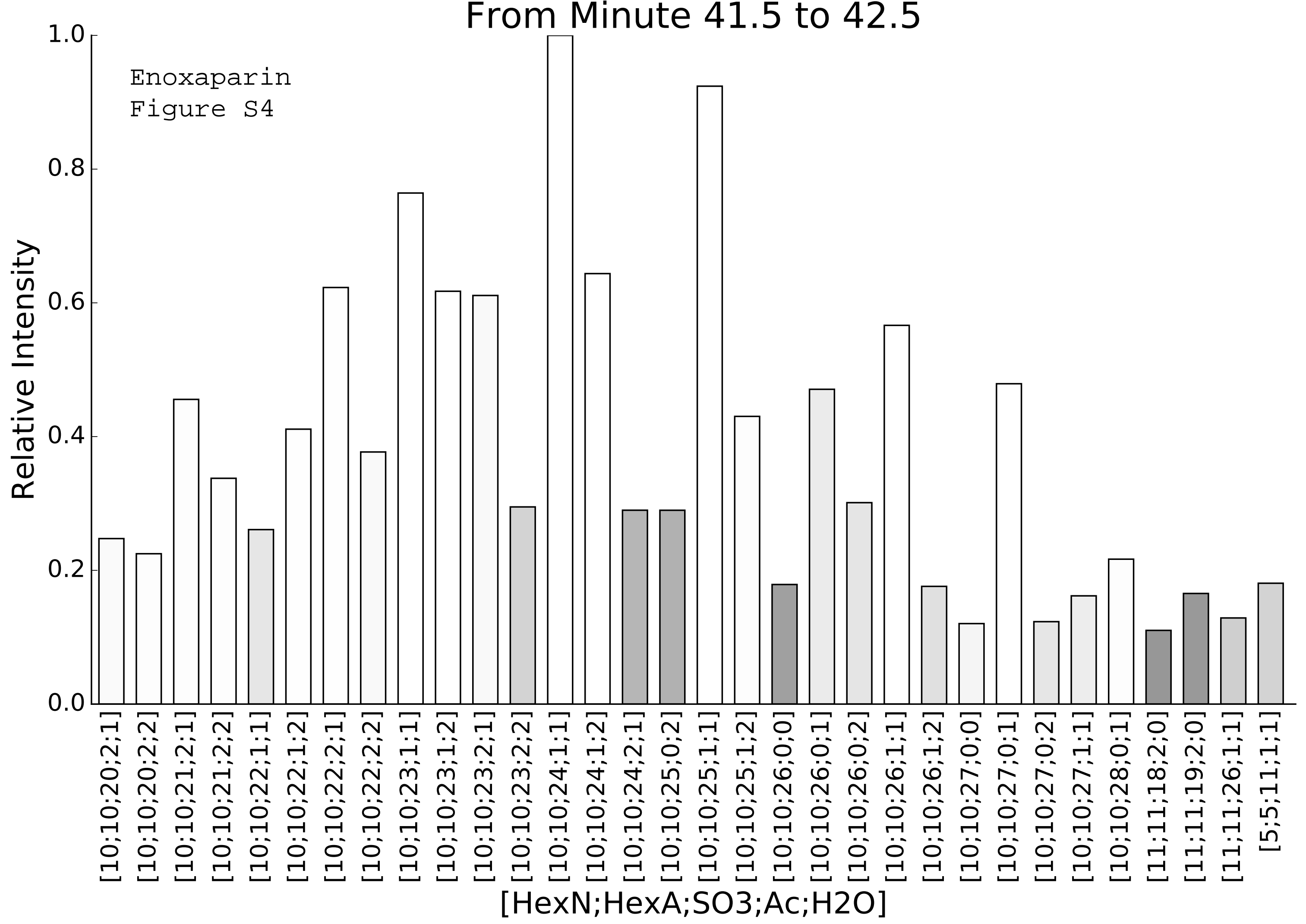




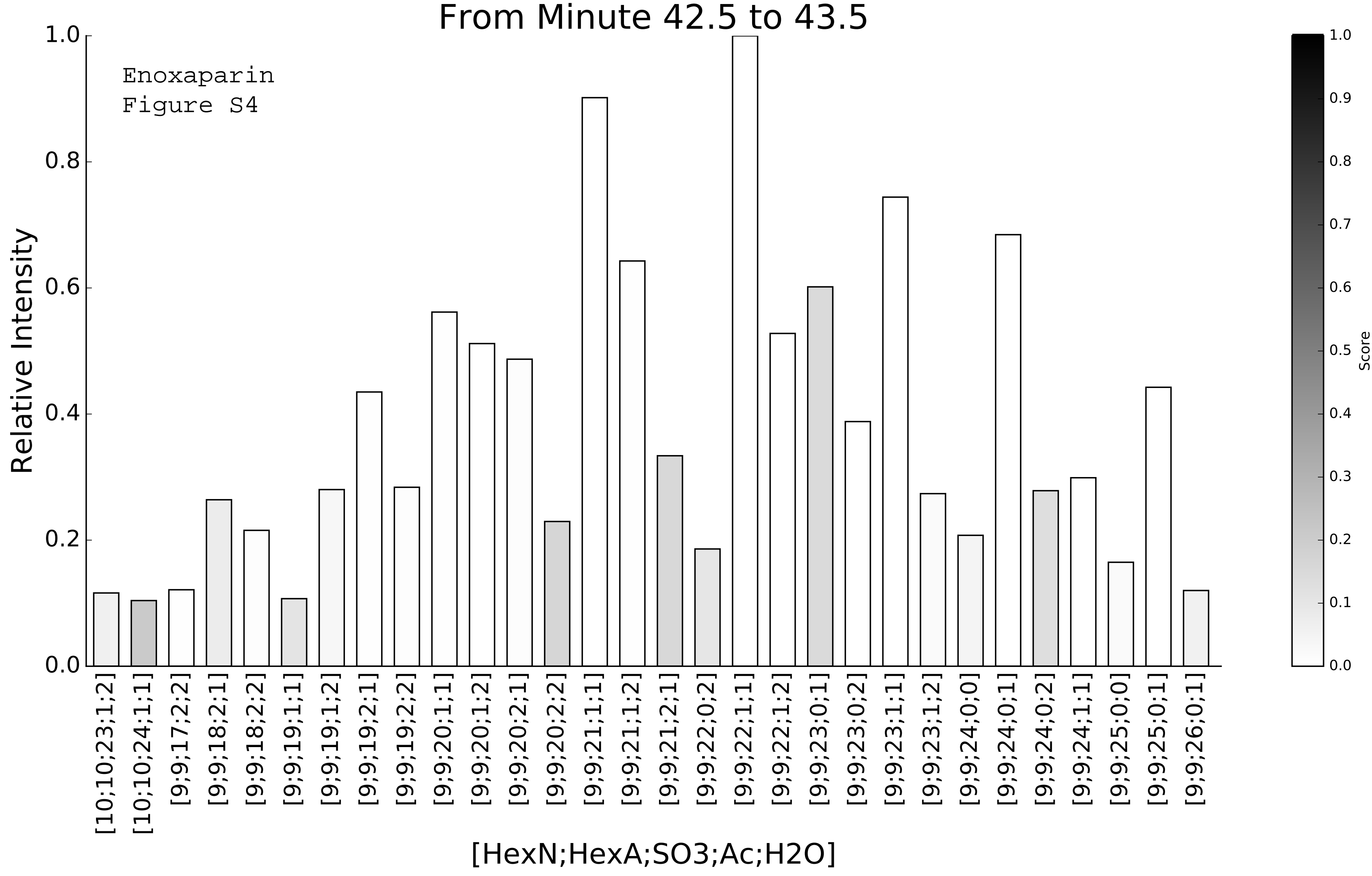




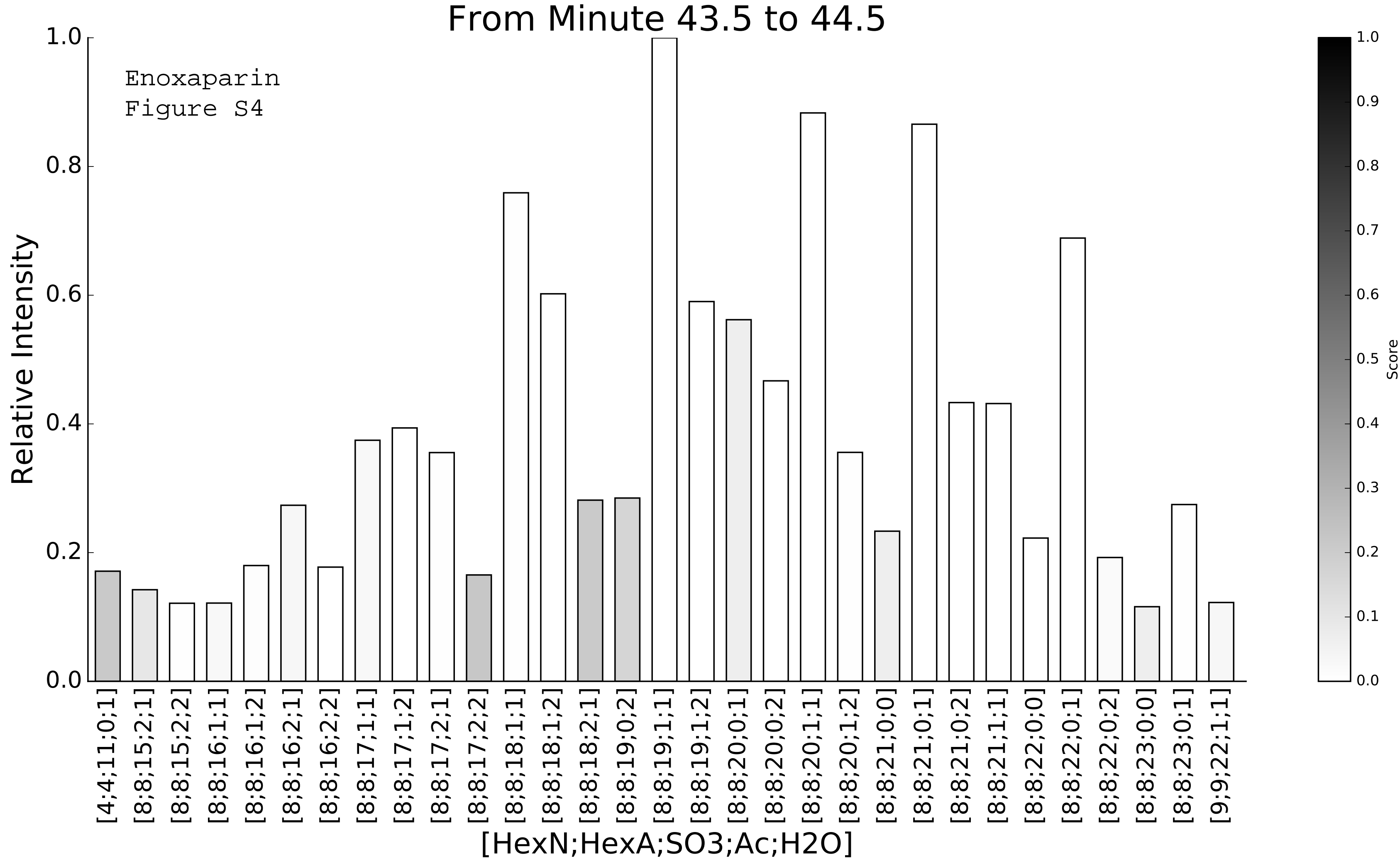




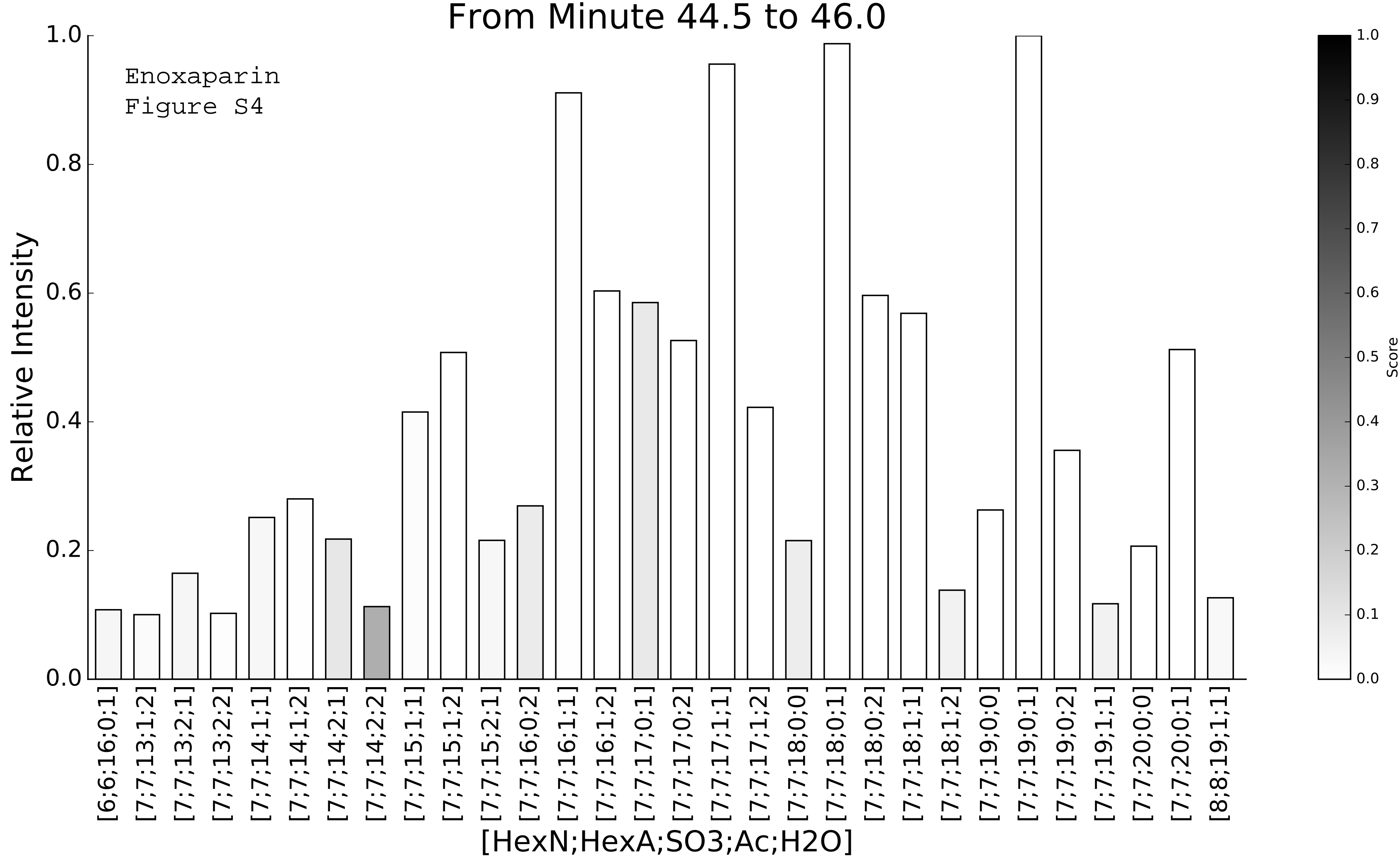




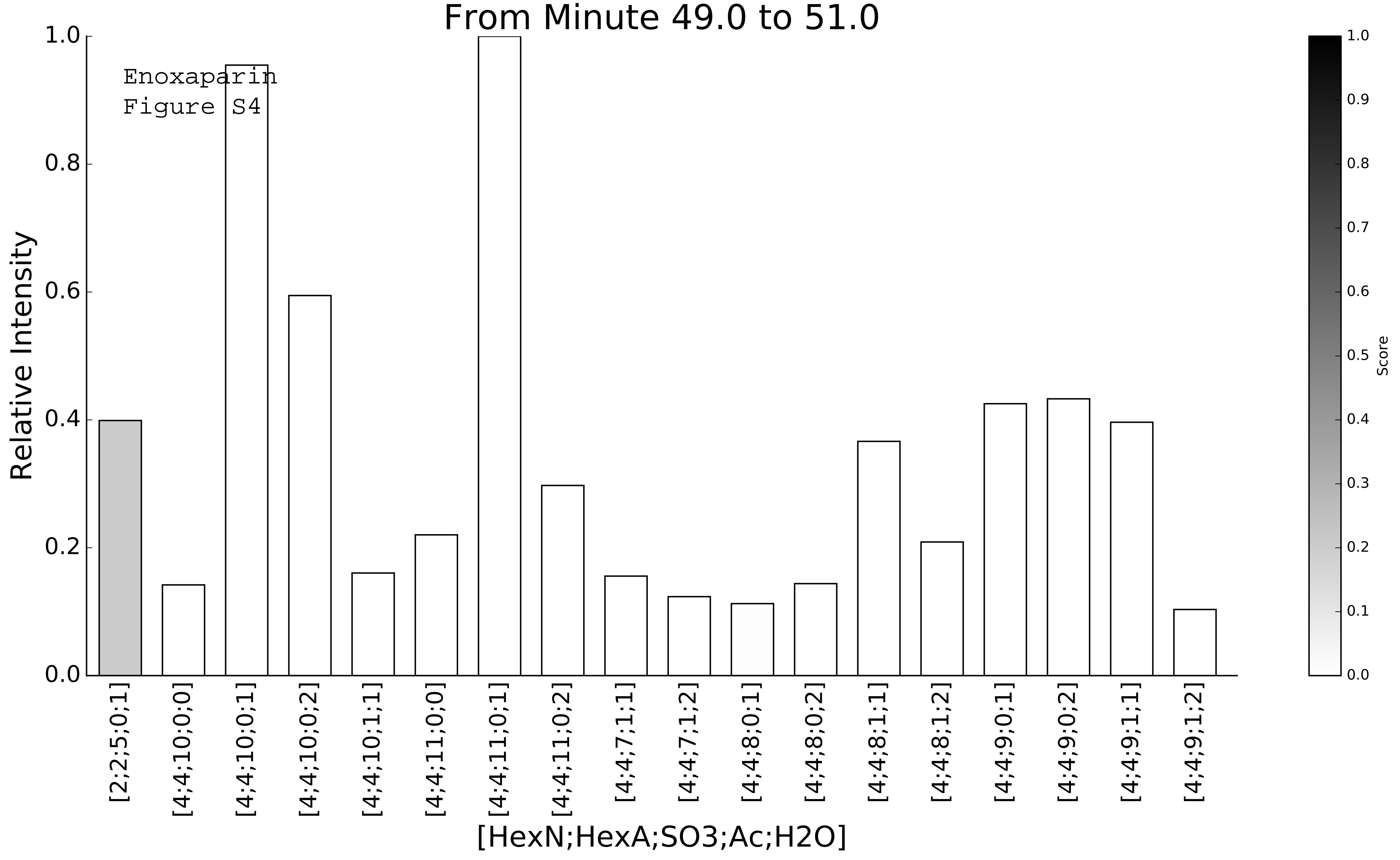




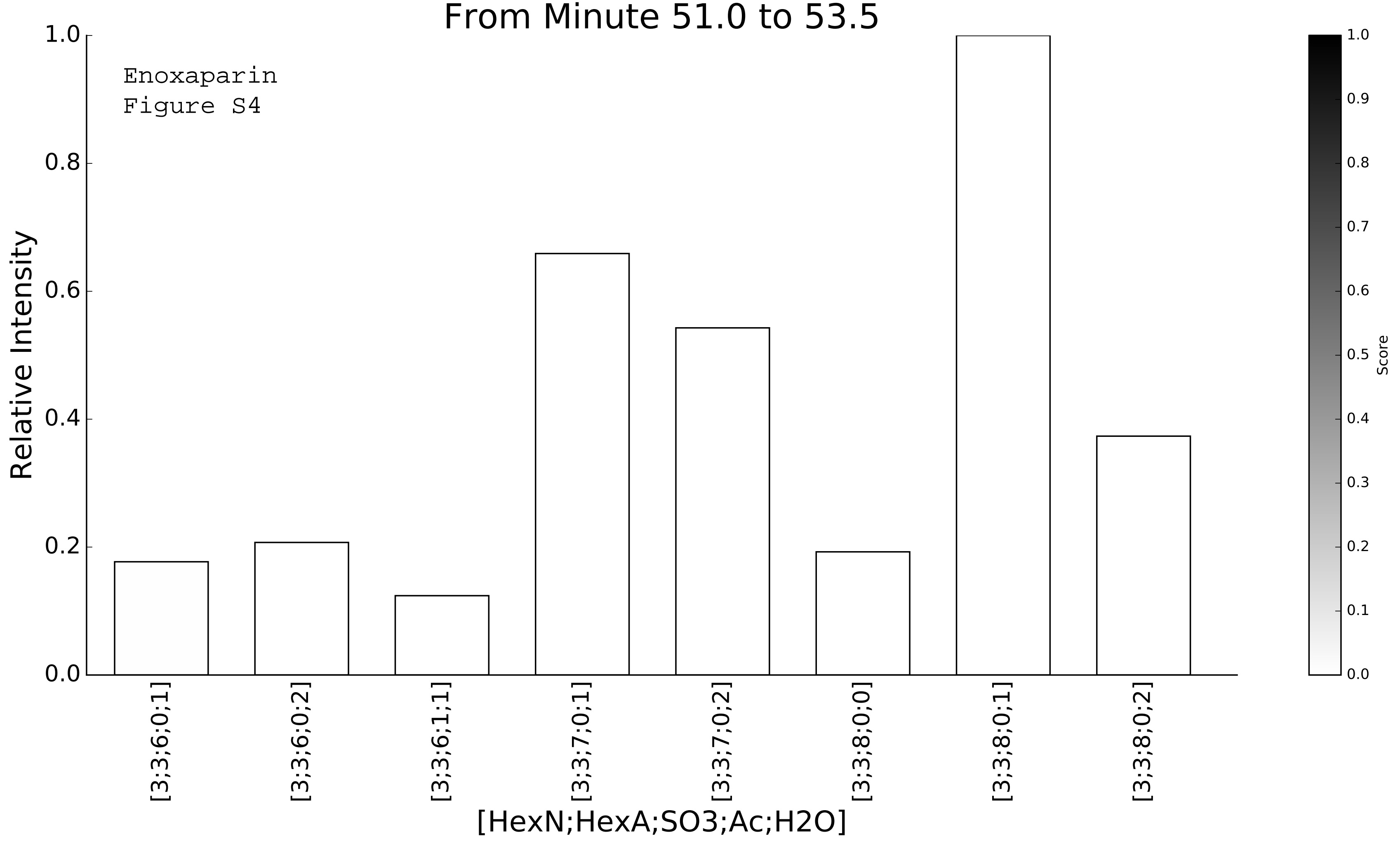


Composition Abundance Across Charge States
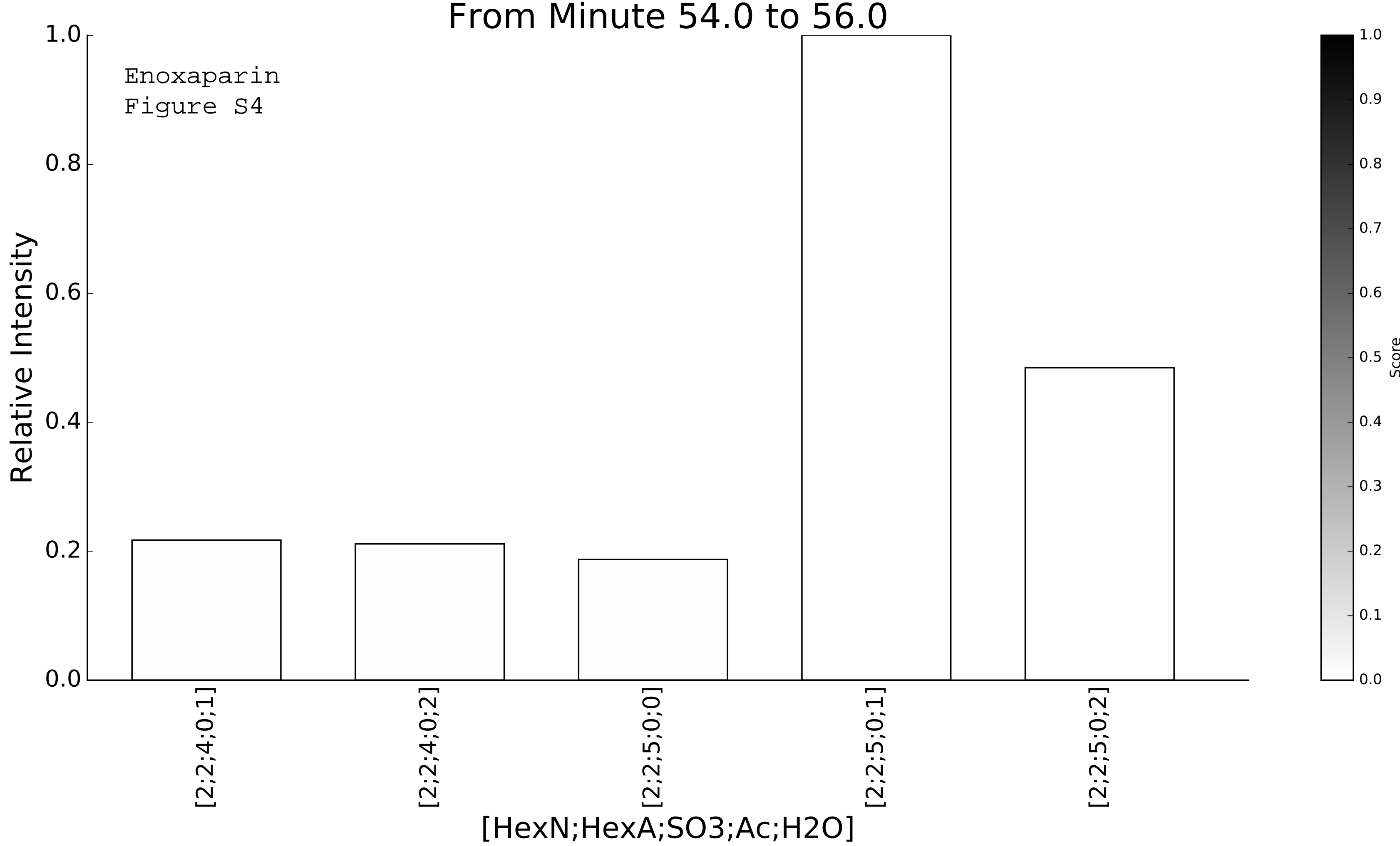
